\title{
Imprint of First Stars Era in the Cosmic Infrared Background Fluctuations
}

\author{
Alexander Kashlinsky \\ SSAI and Observational Cosmology Lab, Code 665, Goddard Space Flight Center, Greenbelt, \\ MD 20771, U.S.A. \\ email: Alexander.Kashlinsky.1@nasa.gov
}

\begin{abstract}
We present the latest results on Cosmic Infrared Background (CIB) fluctuations from early epochs from deep Spitzer data. The results show the existence of significant CIB fluctuations at the IRAC wavelengths $(3.6$ to $8 \mu \mathrm{m})$ which remain after removing galaxies down to very faint levels. These fluctuations must arise from populations with a significant clustering component, but only low levels of the shot noise. There are no correlations between the source-subtracted IRAC maps and the corresponding fields observed with the HST ACS at optical wavelengths. Taken together, these data imply that 1) the sources producing the CIB fluctuations are individually faint with $S_{\nu}<$ a few nJy at 3.6 and $4.5 \mu \mathrm{m} ; 2$ ) have different clustering pattern than the more recent galaxy populations; 3 ) are located within the first 0.7 Gyr (unless these fluctuations can somehow be produced by - so far unobserved - local galaxies of extremely low luminosity and with the unusual for local populations clustering pattern), 4) produce contribution to the net CIB flux of at least $1-2 \mathrm{nW} \mathrm{m}^{-2} \mathrm{sr}^{-1}$ at 3.6 and $4.5 \mu \mathrm{m}$ and must have mass-to-light ratio significantly below the present-day populations, and 5) they have angular density of $\sim$ a few per $\operatorname{arcsec}^{2}$ and are in the confusion of the present day instruments, but can be individually observable with $J W S T$.
\end{abstract}

Keywords. diffuse radiation - early universe - large-scale structure of universe

\section{Introduction}

The cosmic infrared background (CIB) is a repository of emissions throughout the entire history of the Universe. The recent years have seen significant progress in CIB studies, both in identifying and/or constraining its mean level (isotropic component) and fluctuations (see Kashlinsky 2005 for a recent review). The CIB contains emissions also from objects inaccessible to current (or even future) telescopic studies and can, therefore, provide unique information on the history of the Universe at very early times. One particularly important example of such objects, of particular reference to this conference, concerns Population III stars (hereafter Pop III), the still elusive zero-metallicity stars expected to have been much more massive than the present stellar populations (see Bromm \& Larson 2004 for a recent review). Here I will use the term "era of the first stars", or "Pop III era", with the understanding that the actual era may be composed of objects of various nature from purely zero-metallicity stars, to low- metallicity stars to even possibly mini-quasars whose contribution to the CIB is driven by energy released by gravitational accretion, as opposed to stellar nucleosynthesis.

Extensive numerical investigations of collapse and fragmentation of the first objects forming out of density fluctuations specified by the standard $\Lambda$ CDM model suggest that Pop III stars were quite massive and lived at $z>10$, well within the first Gyr of the Universe's evolution. If predominantly massive, they are expected to have left a significant level of diffuse radiation redshifted today into the IR, and it has been suggested that the CIB contains a detectable contribution from Pop III in the near-IR, manifest in both its 
mean level and its anisotropies (e.g. Bond et al. 1986, Santos et al. 2002, Salvaterra \& Ferrara 2003, Cooray et al. 2004, Kashlinsky et al. 2004).

In the past several years a group of us (Kashlinsky, Arendt, Mather \& Moseley 2005, 2007a,b,c - hereafter KAMM1, KAMM2, KAMM3, KAMM4) have used deep-integration Spitzer data to measure the CIB fluctuations component arising from early populations. These provide first observational insights into the global evolution of the Universe at early cosmic epochs. Our measurements revealed significant CIB fluctuations at the IRAC wavelengths $(3.6$ to $8 \mu \mathrm{m}$ ) which remain after removing galaxies down to very faint levels (KAMM1, KAMM2). These fluctuations must arise from populations that have a significant clustering component, but only low levels of the shot noise (KAMM3). Furthermore, there are no correlations between the source-subtracted IRAC maps and the corresponding fields observed with the HST ACS at optical wavelengths (KAMM4). Taken together, these data imply that 1) the sources producing the CIB fluctuations have a very different clustering pattern than galaxies at intermediate redshifts and are individually faint with $S_{\nu}<$ a few nJy at 3.6 and $4.5 \mu \mathrm{m} ; 2$ ) are located within the first $\simeq 0.7$ Gyr (unless these fluctuations can somehow be produced by - so far unobserved - local galaxies of extremely low luminosity and with the unusual for local populations clustering pattern), 3) they produce contribution to the net CIB flux of at least 1-2 $\mathrm{nW} \mathrm{m}^{-2} \mathrm{sr}^{-1}$ at 3.6 and $4.5 \mu \mathrm{m}$ and must have mass-to-light ratio significantly below the present-day populations, and 4 ) their angular density is $\sim($ a few $) \operatorname{arcsec}^{-2}$, so they are in the confusion of the current instruments, but can be individually observable with JWST.

Below, I will discuss the latest measurements of the fluctuations in the CIB by our team (Kashlinsky, Arendt, Mather \& Moseley - KAMM) and explore their implications for the nature of the sources contributing to these anisotropies, specifically in the era of the first stars. Following the Introduction, Sec. 2 reviews the current measurements at both near-IR (IRAC) and optical (ACS) wavelengths and Sec. 3 discusses the nature of the cosmological populations producing these CIB anisotropies.

\section{Source subtracted CIB fluctuations vs optical galaxies}

Before we discuss the interpretation of the KAMM measurements, it is important to review the steps done in the analysis leading to the measured CIB fluctuations. The data have been assembled from the individual AORs using the self-calibration method from Fixsen et al. (2000). The exposure times ranged from $\sim 8-9$ hours/pixel for the initial $5^{\prime} \times 10^{\prime}$ QSO 1700 field (KAMM1) to $\sim 23-25$ hours/pixel in each of the four GOODS fields of $10^{\prime} \times 15^{\prime}$ (KAMM2) The latter have been observed at two different epochs separated by $\sim 6$ months allowing us to better handle zodiacal gradients and possible instrument systematics. The images have been cleaned of foreground galaxies and stars in two steps: 1) all pixels with flux fluctuations exceeding a fixed number of standard deviations of the image were iteratively blanked, and 2) the residual faint parts of the sources were removed iteratively using a modified CLEAN algorithm (Hogbom 1974) where the maximum pixel intensity is located and the wide PSF is then scaled to half of this intensity and subtracted from the image. In the first step, which defines the final mask, it is important to remain in the regime when the fraction of the removed pixels is small enough to allow a reliable computation of the power spectrum using FFTs. The second step allows us removal of progressively fainter foreground populations and enables a better characterization of the remaining (and removed) populations.

Left panels of Fig. 1 show the source-subtracted CIB fluctuations (the instrument noise has been subtracted) for four GOODS fields at 3.6 and $4.5 \mu \mathrm{m}$ adopted from 
KAMM2. The detected signal is significantly higher than the instrument noise and the various systematics effects cannot account for it. Similarly, fluctuations due to emissions in the Solar System and the Galaxy are too weak, except at $8 \mu \mathrm{m}$ where Galactic cirrus may contribute to the measured signal. There was a statistically significant correlation between the channels for the regions of overlap meaning that the same population is responsible for the fluctuations. The correlation function at deeper clipping cuts, when too few pixels were left for Fourier analysis, remains the same and is consistent with the power spectrum numbers (KAMM1). The signal is to a good accuracy isotropic on the sky, as required by its extragalactic origin, and must thus contain contributions from the "ordinary" galaxies and from unresolved populations at high $z$.

The extragalactic signal is made of two components: 1) shot noise from the remaining faint galaxies (shown with dotted lines in Fig. 1), and 2) on arcminute scales the fluctuations are produced by clustering of the emitters. It is important to emphasize that as fainter foreground galaxies are removed so that the remaining shot noise is reduced the details of the fluctuations change. This is due to the varying contribution from the remaining foreground galaxies. The large-scale part of the fluctuations remains as the foreground sources are removed down to the lowest shot-noise levels. Left panels of Fig. 2 show the decrease in the shot-noise power, $P_{\mathrm{SN}}$, as progressively higher iterations in the source removal are reached. The final shot noise reached by us with the GOODS data is shown with horizontal lines and is a factor of $\sim 2$ lower than in the QSO 1700 data (KAMM1). The right panels of Fig. 2 compare the final shot noise with that produced by the observed galaxy counts (which at these wavelengths are confusion limited at $m_{\mathrm{AB}}>21-22$ for IRAC beam). The figure shows that galaxy removal is efficient to $m_{\mathrm{AB}}>26-27$ and the signal in Fig. 1 comes from very faint sources.
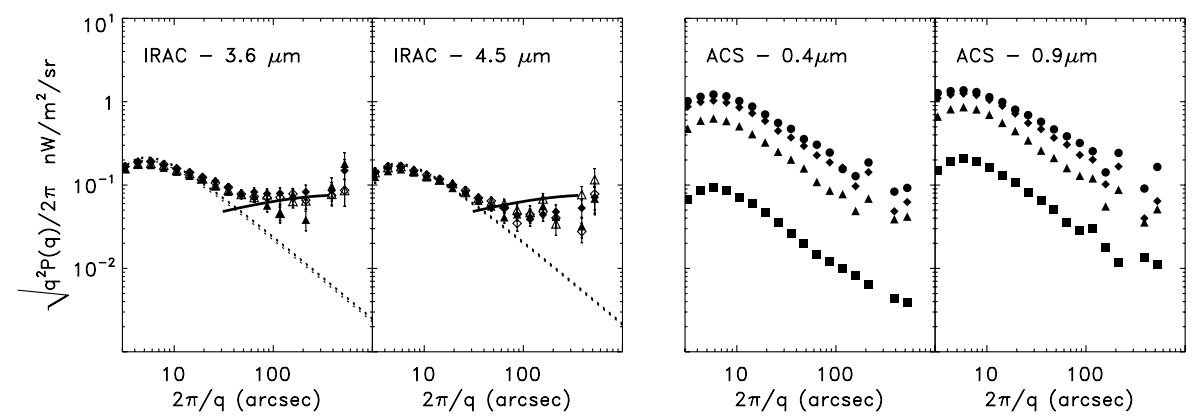

Figure 1. Left: Source-subtracted CIB fluctuations from KAMM2 at 3.6 and $4.5 \mu \mathrm{m}$. Four sets of symbols correspond to the four GOODS fields. Dotted lines show the shot-noise contribution. Solid line shows the slope of sources at high- $z$ with the $\Lambda$ CDM model spectrum of the same amplitude at 3.6 and $4.5 \mu \mathrm{m}$. Right: CIB fluctuations due to ACS galaxies for the $972 \times 9720.6^{\prime \prime}$ pixel field at HDFN-Epoch2 region for the ACS B and z-bands. Filled circles correspond to ACS galaxies fainter than $m_{0}=21$ with the mask defined by the clipping. Filled diamonds, triangles and squares correspond to fluctuations produced by sources fainter than $m_{0}+2, m_{0}+4, m_{0}+6$.

GOODS fields have also been observed at optical wavelengths with the Hubble ACS instruments reaching source detection levels fainter than $28 \mathrm{AB}$ mag. This allowed us to further test the origin of the source-subtracted CIB fluctuations. If the latter come from local populations there should be a strong correlation between the source-subtracted IRAC maps and the ACS sources. Conversely, there should be no such correlations if the CIB signal arises at at epochs where the Lyman break (at rest $\sim 0.1 \mu \mathrm{m}$ ) gets shifted passed the longest ACS z-band at $\simeq 0.9 \mu \mathrm{m}$. To test for this in KAMM4 we have constructed synthetic maps, overlapping with the GOODS fields, using sources in the ACS 

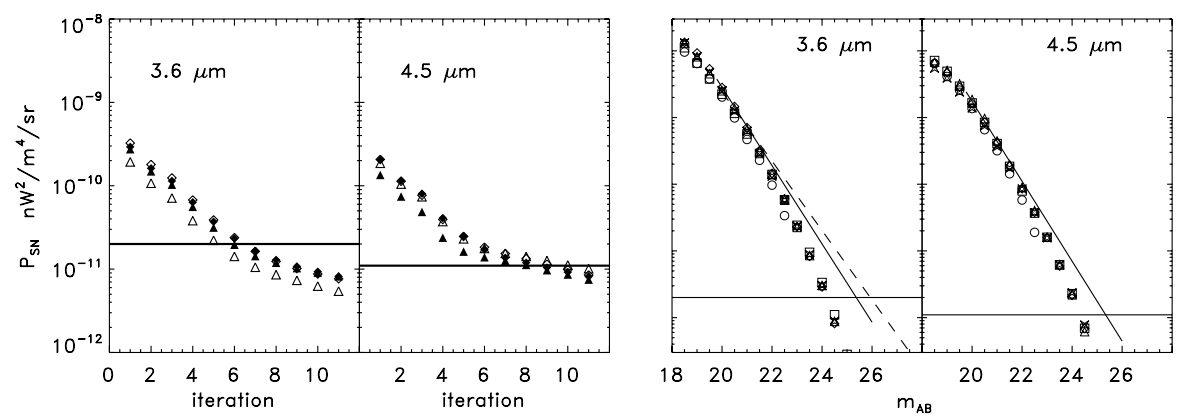

Figure 2. Left: Decrease of the shot noise power vs the iteration number of the source cleaning. Horizontal lines show the levels reached in Kashlinsky et al. (2007), which are a factor of $\sim 2$ below those in Kashlinsky et al. (2005). Four sets of symbols correspond to the four GOODS fields. Horizontal lines mark the shot noise levels of KAMM2. Right: Shot noise power $P_{\mathrm{SN}}$ estimated by integrating the counts. Symbols identical to those on the left denote the four GOODS fields; open circles correspond to counts for the QS1700 field. Solid line shows $P_{\mathrm{SN}}$ according to the fit to IRAC counts of Fazio et al. (2004) used in KAMM1.

B, V, i, z bands from the ACS sources catalog of Giavalisco et al. (2004). These maps were then convolved with the IRAC 3.6 and $4.5 \mu \mathrm{m}$ beams. Finally, we applied the clipping mask from the IRAC maps and computed the fluctuations spectrum produced by the ACS sources and their correlations with the IRAC-based maps.

The fluctuations in the diffuse light produced by the ACS galaxies are shown in the right panels of Fig. 1. The contrast between the spectrum of the source-subtracted CIB fluctuations and those produced by the optical galaxies is obvious. The former has the power spectrum such that the fluctuations are flat to slowly rising with increasing angular scale, whereas the latter have power spectrum with fluctuation amplitude decreasing with increasing scale in agreement with CIB measurements from deep 2MASS data arising from galaxies at $z \sim 1-2$ (Kashlinsky et al 2002). This by itself shows that the populations producing the source-subtracted CIB fluctuations are not in the ACS source catalog.

More importantly, Fig. 3 shows that the correlations between the ACS galaxies and the source-subtracted CIB maps are very small and on arcminute scales are within the statistical noise. Thus, at most, the remaining ACS sources contribute to the shot-noise levels in the residual KAMM maps, but not to the large scale correlations. At the same time, there are excellent correlations (shown with open symbols) between the ACS source maps and the sources removed by KAMM prior to computing the remaining CIB fluctuations.

The excess source-subtracted CIB fluctuation on arcminute scales in the $3.6 \mu \mathrm{m}$ channel is $\sim 0.1 \mathrm{nW} \mathrm{m}^{-2} \mathrm{sr}^{-1}$; KAMM measure a similar amplitude in the longer IRAC bands indicating that the energy spectrum of the arcminute scale fluctuations is flat to slowly rising with increasing wavelength at least over the IRAC range of wavelengths. This is illustrated with the solid line in the left panels of Fig. 1.

\section{Cosmological implications}

Any interpretation of the KAMM results must reproduce three major aspects:

- The sources in the KAMM data were removed to a certain (faint) flux limit, so the CIB fluctuations arise in populations with magnitudes fainter than the corresponding magnitude limit, $m_{\text {lim }}$. Furthermore, these sources are not present among the optical ACS galaxies as demonstrated by the absence of correlations between these galaxies and the IRAC source-subtracted CIB maps. 

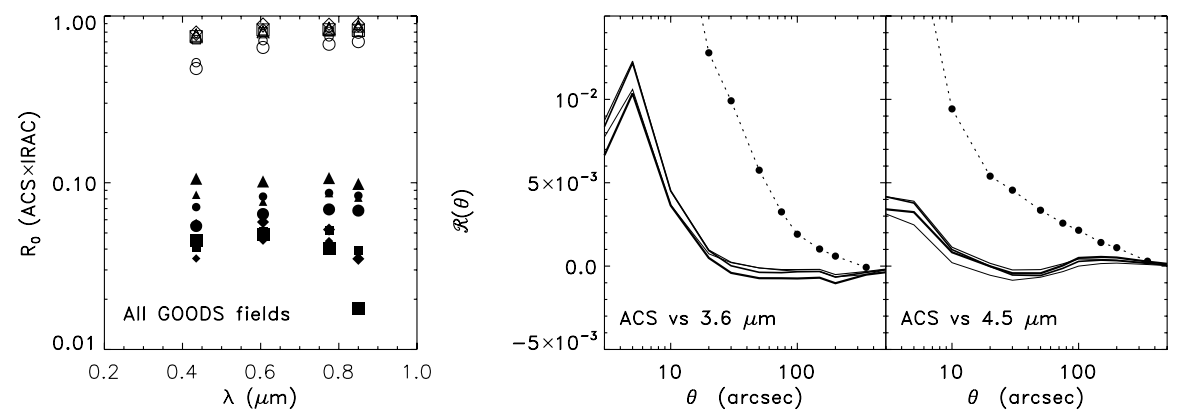

Figure 3. Left: Correlation coefficient between clipped/masked ACS and KAMM data. Large and small symbols correspond to the IRAC Ch 1 and $\mathrm{Ch} 2$; the four sets of symbols correspond to the four GOODS fields. Open symbols correspond to correlations with the maps of the removed sources and filled symbols with the residual KAMM maps which contain the fluctuations shown in Fig. 1. Right: Solid lines show the dimensionless correlation function between the diffuse light in the ACS and KAMM maps for $B, V, i, z$-bands in order of increasing thickness. Dotted line shows the dimensionless correlation function of the KAMM maps, $C_{\mathrm{KAMM}}(\theta) / \sigma_{\mathrm{KAMM}}^{2}$, which remains positive out to $\sim 100^{\prime \prime}$ and is better viewed in log-log plots as in Fig. SI-4 of KAMM1.

- These sources must reproduce the excess CIB fluctuations by KAMM on scales $>0.5^{\prime}$. They must also reproduce the measured spectrum of the CIB fluctuations, which is different from the observed clustering pattern of ordinary galaxies at intermediate $z$.

- Lastly, the populations fainter than the above magnitude limit must account not only for the correlated part of the CIB, but - equally important - they must reproduce the (low) shot-noise component of the KAMM signal, which dominates the power at $<0.5^{\prime}$. The discussion below by-and-large follows KAMM3:

1) Magnitude limits and epochs. Since the ACS galaxies do not contribute to the source-subtracted CIB fluctuations, the latter must arise at $z>7$ as is required by the Lyman break at rest $\sim 0.1 \mu \mathrm{m}$ getting redshifted past the ACS $z$-band of peak wavelength $\simeq 0.9 \mu \mathrm{m}$. This would place the sources producing the KAMM signal within the first 0.7 Gyr. If the KAMM signal were to originate in lower $z$ galaxies which escaped the ACS GOODS source catalog because they are below the catalog flux threshold, they would have to be extremely low-luminosity systems $\left(<2 \times 10^{7} h^{-2} L_{\odot}\right.$ at $\left.z=1\right)$ and these galaxies would also have to cluster very differently from their ACS counterparts.

2) Clustering component. Solid lines in Fig. 1 show the expected CIB fluctuations from sources with the (biased) concordance $\Lambda$ CDM power spectrum at $z>5$. The fit is reasonably good making such sources a plausible candidate for producing the observed signal. At the same time, the observed galaxy populations out to $z \sim 2-3$ cluster very differently. Thus any model attempting to assign the KAMM signal to more recent sources will have to account for this observed difference in the clustering patterns.

3) Net CIB levels from the new sources: The angle of $1^{\prime}$ in the concordance cosmology subtends comoving scales of $2.2-3 \mathrm{Mpc}$ at $5 \leqslant z \leqslant 20$. For $\Lambda$ CDM density fields with reasonable biasing one can reach relative arcminute-scale fluctuations of $\sim 5-10 \%$ meaning that the net CIB from sources contributing to the KAMM signal at $3.6 \mu \mathrm{m}$ is at least $1-2 \mathrm{nW} \mathrm{m}^{-2} \mathrm{sr}^{-1}$, which is well within the uncertainties of the recent CIB measurements of Thompson et al. $(2007 \mathrm{a}, \mathrm{b})$.

4) Shot noise constraints. The amplitude of the shot-noise power gives a particularly strong indication of the epochs of the sources contributing to the KAMM signal. This 
can be seen from the expressions for the shot noise (Kashlinsky 2005a):

$$
P_{\mathrm{SN}}=\int_{m_{\mathrm{AB}}>m_{\mathrm{lim}}} f(m) d F(m) \equiv f(\bar{m}) F_{\mathrm{tot}}\left(m_{\mathrm{AB}}>m_{\mathrm{lim}}\right)
$$

where $f(m)$ is the flux in Jy of a source of magnitude $m$ and $F_{\text {tot }}\left(m_{\mathrm{AB}}>m_{\mathrm{lim}}\right)$ is the net CIB flux produced by the remaining sources. Above it was shown that the sources contributing to the fluctuations must have CIB flux greater than a few $\mathrm{nW} \mathrm{m}^{-2} \mathrm{sr}^{-1}$ and combining this with the values for $P_{\mathrm{SN}} \sim 10^{-11} \mathrm{nW}^{2} \mathrm{~m}^{-4} \mathrm{sr}^{-1}$, reached in the KAMM2 analysis, leads via eq. 3.1 to these sources having typical magnitudes $m_{\mathrm{AB}}<29-30$ or individual fluxes $<4 \mathrm{nJy}$. Such faint sources are expected to lie at very high $z$.

5) Mass/light ratio of the new populations: This information on the nature of the populations responsible for these CIB fluctuations, can be obtained from the fact that the significant amount of flux $\left(>1-2 \mathrm{nW} \mathrm{m}^{-2} \mathrm{sr}^{-1}\right)$ required to explain the amplitude of the fluctuations must be produced within the short time available at these high $z$ (cosmic times $<0.5-1$ Gyr). The implied comoving luminosity density associated with these populations is related to the fraction of baryons locked in these objects with the additional assumption of their $\Gamma \equiv M / L$. The smaller the value of $\Gamma$, the fewer baryons are required to explain the CIB fluctuations detected in the KAMM studies. It turns out that in order not to exceed the baryon fraction observed in stars, the populations producing these CIB fluctuations had to have $\Gamma$ much less than the solar value, typical of the present-day populations (KAMM3). This is consistent with the general expectations of the first stars being very massive.

6) Resolving the new sources: In order to directly detect the faint sources responsible for the CIB fluctuations with fluxes below a few nJy, their individual flux must exceed the confusion limit. If such sources were to contribute to the CIB required by KAMM data, at 3.6 and $4.5 \mu \mathrm{m}$ they had to have the average surface density of $\bar{n} \sim F_{\mathrm{CIB}}^{2} / P_{\mathrm{SN}} \sim 5 \operatorname{arcsec}^{-2}$. In order to avoid the confusion limit and resolve these sources individually at, say, 5 -sigma level $(\alpha=5)$ one would need a beam of the area $\omega_{\text {beam }} \leqslant \alpha^{-2} / \bar{n} \sim 5 \times 10^{-3} \operatorname{arcsec}^{2}$ or of circular radius below $\sim 0.04$ arcsec. This is clearly not in the realm of the currently operated instruments, but the JWST could be able to resolve these objects given its sensitivity and resolution.

\section{Acknowledgements}

I thank my collaborators, Rick Arendt, John Mather and Harvey Moseley for many contributions to the KAMM results, and the NSF AST-0406587 grant for support.

\section{References}

Bond, J. R., Carr, B. J., \& Hogan, C. J. 1986, ApJ, 306, 428

Bromm, V. \& Larson, R. 2004, ARA\&A, 42, 79

Cooray, A., Bock, J. J., Keatin, B., et al. 2004, ApJ, 606, 611

Fazio, G., Ashby, M. L. N., Barnby, P., et al. 2004, ApJS, 154, 39

Fixsen, D., Moseley, S. H. \& Arendt, R. G. 2000, ApJS, 128, 651

Giavilisco, M., Dickinson, M., Ferguson, H. C., et al. 2004, ApJ, 600, L93

Hogbom, J. 1974, A\&AS, 15, 417

Kashlinsky, A. 2005, Phys. Rep., 409, 361

Kashlinsky, A. 2005, ApJ, 633 L5

Kashlinsky, A., Odenwald, S., Mather, J., et al. 2002, ApJ, 579, L53

Kashlinsky, A., Arendt, R. G., Gardner, J. P., et al. 2004, ApJ, 608, 1

Kashlinsky, A., Arendt, R. G., Mather, J., \& Moseley, S. H. 2005, Nature, 438, 45 (KAMM1)

Kashlinsky, A., Arendt, R. G., Mather, J., \& Moseley, S. H. 2007a, ApJ, 654, L5 (KAMM2) 
Kashlinsky, A., Arendt, R. G., Mather, J., \& Moseley, S. H. 2007b, ApJ, 654, L1 (KAMM3)

Kashlinsky, A., Arendt, R. G., Mather, J., \& Moseley, S. H. 2007c, ApJ, 666, L1 (KAMM4)

Santos, M., Bromm, V., \& Kamionkowski, M. 2002, MNRAS, 336, 1082

Thompson, R., Eisenstein, D., Fan, X., et al. 2007a, ApJ, 659, 667

Thompson, R., Eisenstein, D., Fan, X., et al. 2007b, ApJ, 666, 658

\section{Discussion}

FynBO: In Chary et al. (2007, ApJL submitted, ArXiv:0711.4099) a significant fraction of the fluctuation signal is associated with low luminosity galaxies at $\langle z\rangle=2.5$. Could you please comment on that?

KASHLINSKY: We have demonstrated in Kashlinsky et al. (2007c) that there are completely negligible correlations between the ACS galaxies, such as considered by Chary et al., and our source-subtracted IRAC maps from which we derive the CIB fluctuations at $3.6 \mu \mathrm{m}$ and $4.5 \mu \mathrm{m}$. This means that the ACS-detected galaxies contribute negligibly to the CIB fluctuations measured by us. Additionally, the clustering pattern of the ACS galaxies is such that their diffuse light fluctuations decrease strongly with angular scale, whereas our CIB fluctuations are flat to slowly rising with increasing angular scale. This further demonstrated that populations producing the source-subtracted CIB fluctuations are of a different nature than those in the ACS measurements.

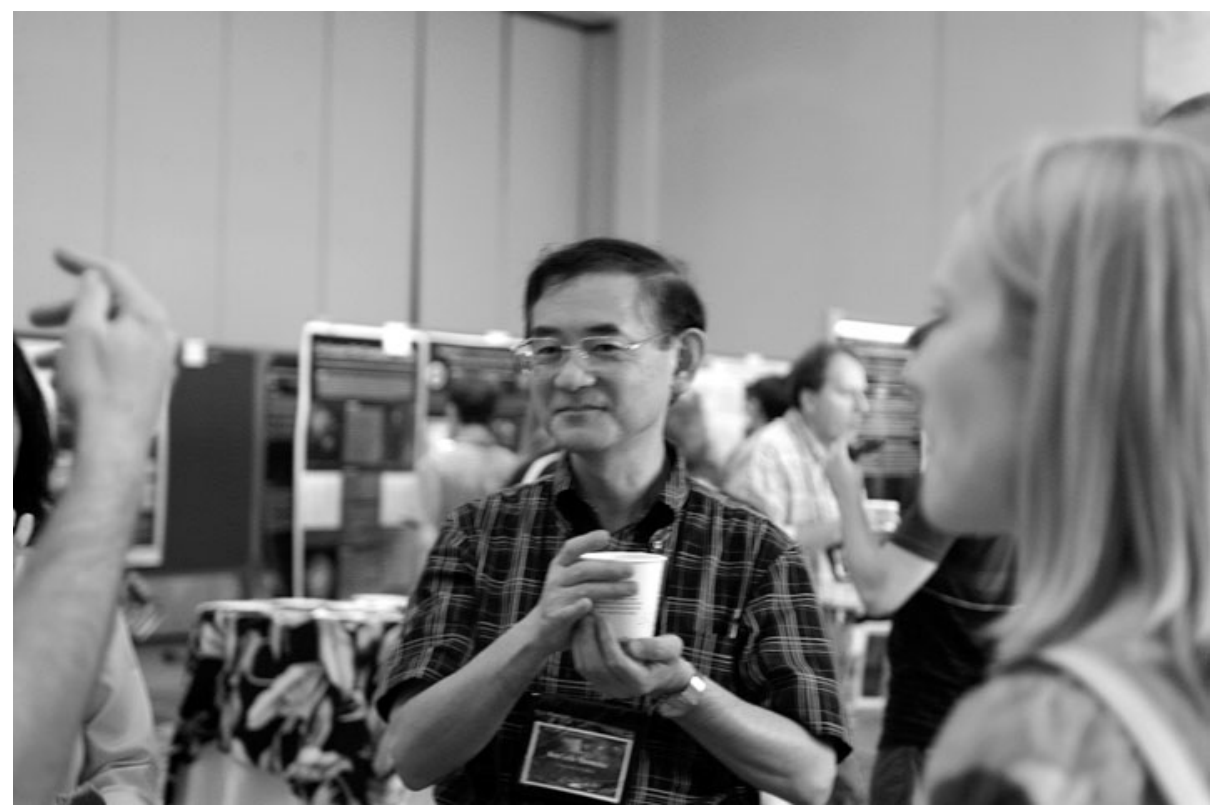

Ken Nomoto. 


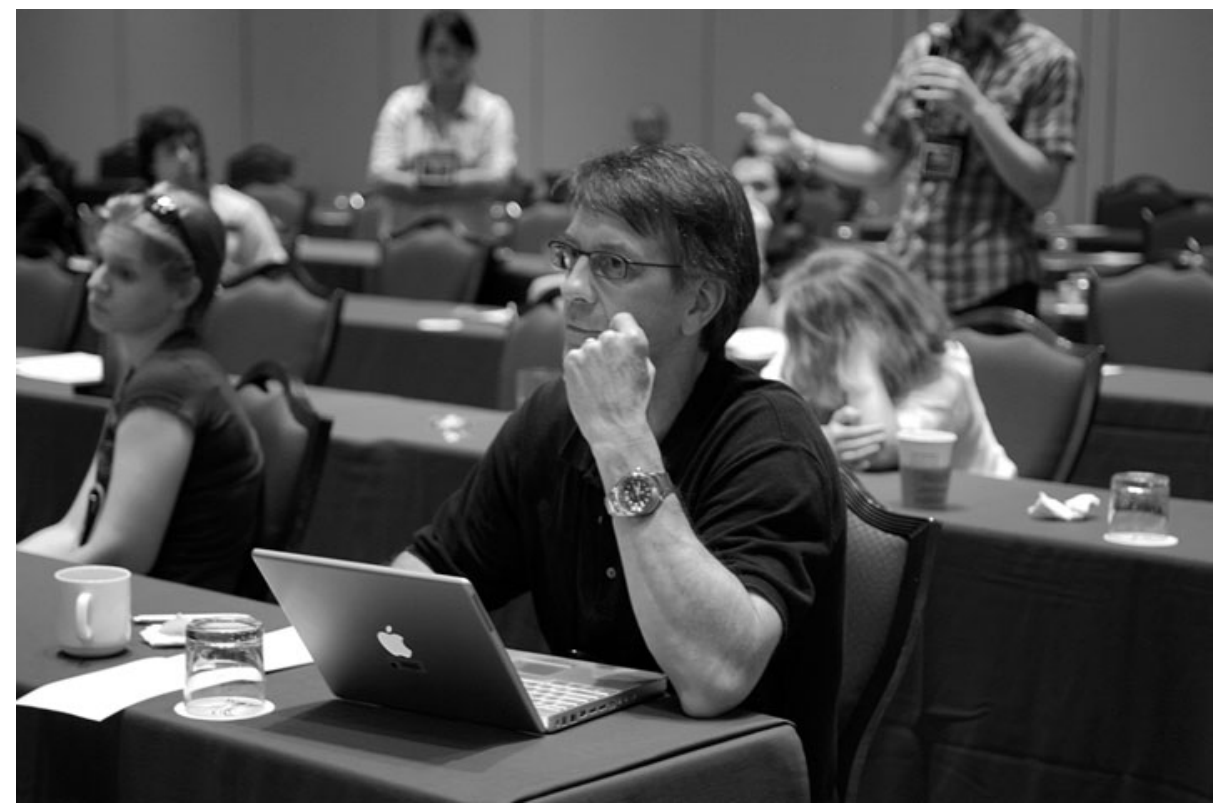

George Sonneborn.

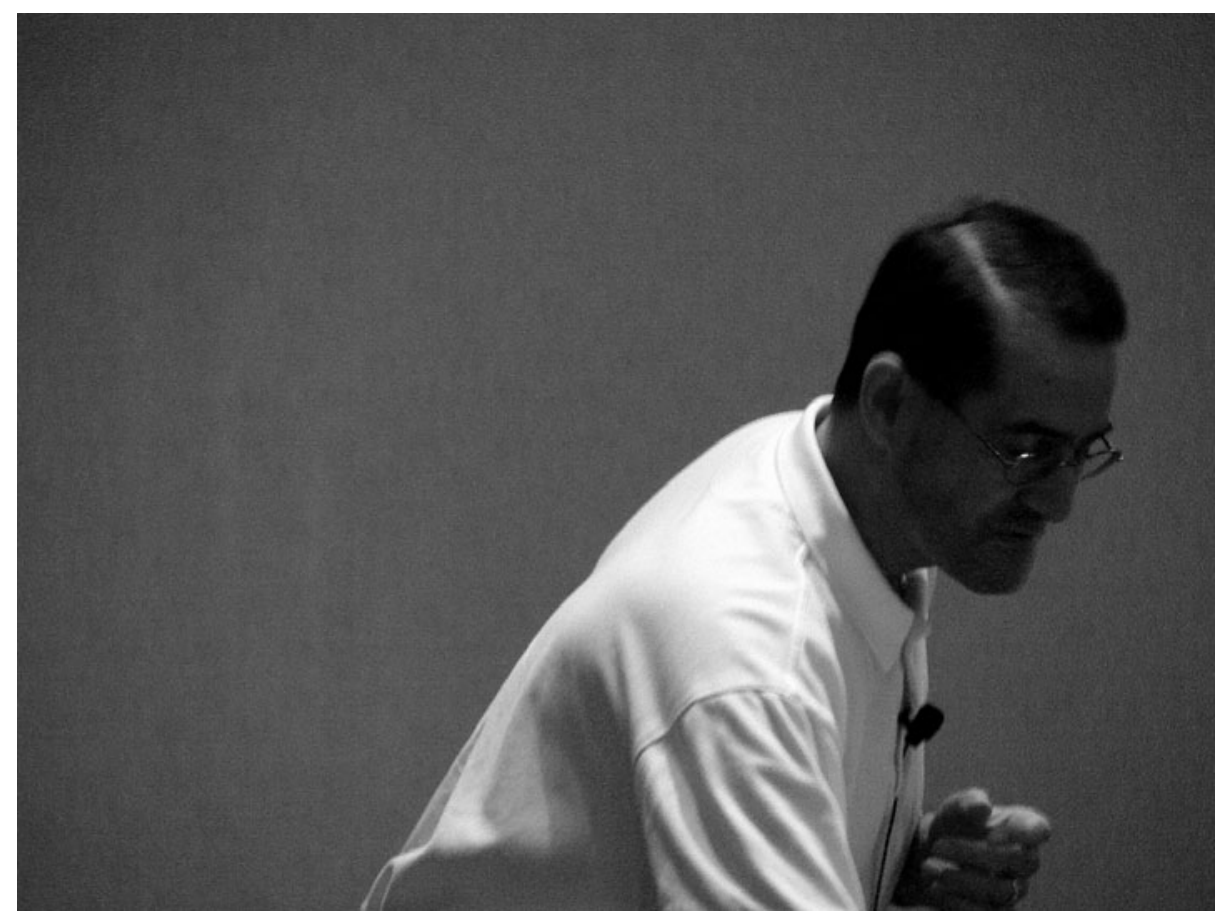

Claus Leitherer. 